\title{
Studies in Oxadiazoles \\ Synthesis of Some 2-Mercapto-1,3,4-oxadiazoles and Related Compounds as Potential Fungicides
}

\author{
S. GIRI, H. SINGH and L. D. S. YADAV \\ Chemistry Department, University of Gorakhpur, Gorakhpur (U.P.) India
}

Received May 14, 1975

\begin{abstract}
Several 2-mercapto-5-substituted-1,3,4-oxadiazoles, alkyl and aralkyl oxadiazolyl sulphides, disulphides and sulphones have been prepared. A representative number of these compounds have been tested against two fungal species.
\end{abstract}

Although the oxadiazole ring is associated with many types of biological properties such as antifungal, ${ }^{1)}$ hypoglycemic, ${ }^{2)}$ analgesic ${ }^{32}$ and herbicidal ${ }^{4}$ etc., the fungicidal properties of the oxadiazole sulphones and related compounds have not been apparently studied. Since a number of aryl and heteroaryl sulphones display pesticidal ${ }^{5 \sim 8)}$ tuberculostatic ${ }^{99}$ and antistreptococcal ${ }^{10)}$ properties, it was presumed that the sulphones and sulphides derived from biologically versatile oxadiazoles might be the fungicides of choice. Further, the mercapto oxadiazoles from which the above compounds are derived might themselves be useful fungicides since the - $\mathrm{SH}$ group attached to the heterocyclic nucleus may induce fungicidal action. ${ }^{11 \text { ? }}$

Keeping above facts in view, we considered it of interest to synthesise these oxadiazoles and study their fungicidal properties. The 2-mercapto-5-substituted oxadiazoles were prepared by reacting acid hydrazides with carbon disulphide in alkaline medium following the method of Young and Wood. ${ }^{12}$ Reaction of mercapto oxadiazole with alkyl and aralkyl halides $^{13,14)}$ in basic medium gave the corresponding sulphides. The oxidation of these sulphide with hydrogen peroxide by a procedure similar to that adopted by Bambas ${ }^{10)}$ furnished the desired sulphones. The disulphides were obtained by oxidation of the sulphides with bromine. ${ }^{14)}$

A representative number of each type of oxadiazole was tested against two fungal species viz. A. niger and A. flavus. All compounds are toxic to both the species at higher concentrations but their toxicity decreases on dilution. The oxadiazoles with 2-mercapto substituent appear to be more toxic to A. niger compared to A. flavus. Similarly the sulphides are relatively less toxic than sulphones. In all such compounds the chloro- and nitrosubstituents in aryl moiety appear to enhance the fungitoxicity.

\section{EXPERIMENTAL}

2-Mercapto-5-substituted-1, 3, 4-oxadiazoles. These were prepared according to the method of Young and Wood ${ }^{12)}$ by reacting acid hydrazides $(0.05 \mathrm{M})$ with carbodisulphide $(1 \mathrm{M})$ in presence of ethanolic $\mathrm{KOH}$. The compounds thus prepared are recorded in Table I.

Methyl-(5-substituted-1,3,4-oxadiazol-2-yl) sulphides. A reaction mixture containing equimolecular amounts of methyl iodide and 2-mercapto-5-substituted oxadiazole in ethanolic $\mathrm{KOH}$ was refluxed for $2 \mathrm{hr}$. This was cooled and poured into water. The precipitated product was filtered, washed and recrystallised from ethanol. The compounds thus prepared are recorded in Table II.

Benzyl-(5-substituted-1,3,4-oxadiazol-2-yl) sulphides. Benzyl chloride $(0.02 \mathrm{M})$ and 2-mercapto-5-substitutedoxadiazole $(0.02 \mathrm{M})$ were taken in benzene $(200 \mathrm{ml})$. To this was added triethylamine $(0.02 \mathrm{M})$ and the mixture was refluxed for $2 \sim 3 \mathrm{hr}$. After cooling, the reaction mixture was poured into water, the benzene layer was separated and concentrated to a small bulk when the required product slowly crystallised out. The sulphides thus prepared are recorded in Table II.

Methyl-(5-phenyl-1,3,4-oxadiazol-2-yl) sulphone. Methyl-(5-phenyl-1, 3, 4-oxadiazol-2-yl) sulphide (2 g) 
TABLE I. 2-MERCAPTO-5-SUBSTITUTED-1,3,4-OXADIAZOLES

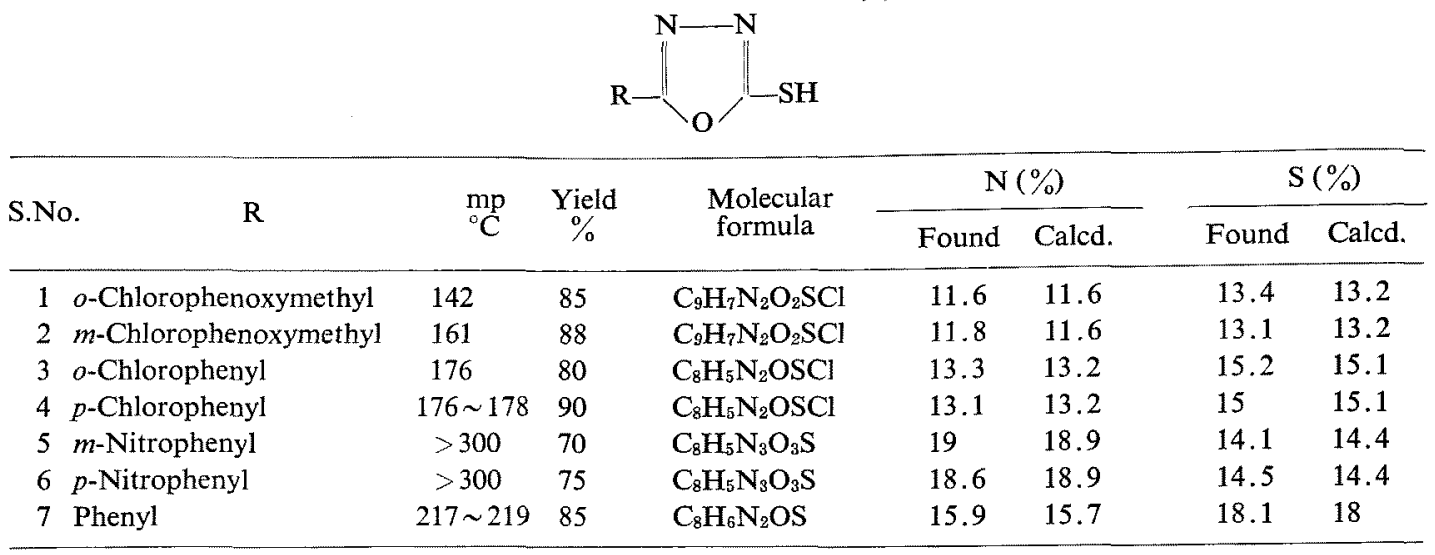

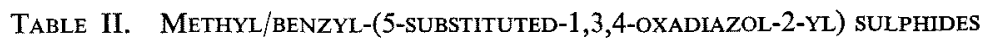<smiles>[R]C1=NN=[SH]([R])O1</smiles>

\begin{tabular}{|c|c|c|c|c|c|c|c|c|}
\hline \multirow{2}{*}{ S.No. } & \multirow{2}{*}{$\mathrm{R}$} & \multirow{2}{*}{${ }^{\circ} \mathrm{Cp}$} & \multirow{2}{*}{$\underset{\%}{\text { Yield }}$} & \multirow{2}{*}{$\begin{array}{l}\text { Molecular } \\
\text { formula }\end{array}$} & \multicolumn{2}{|c|}{$\mathrm{N}(\%)$} & \multicolumn{2}{|c|}{$\mathrm{S}(\%)$} \\
\hline & & & & & Found & Calcd. & Found & Calcd. \\
\hline \multicolumn{9}{|c|}{$\mathrm{R}^{\prime}=$ Methyl } \\
\hline 1 & $o$-Chlorophenoxymethyl & 32 & 66 & $\mathrm{C}_{10} \mathrm{H}_{9} \mathrm{~N}_{2} \mathrm{O}_{2} \mathrm{SCl}$ & 11.1 & 10.9 & 12.3 & 12.5 \\
\hline 2 & $m$-Chlorophenoxymethyl & 58 & 73 & $\mathrm{C}_{10} \mathrm{H}_{9} \mathrm{~N}_{2} \mathrm{O}_{2} \mathrm{SCl}$ & 10.7 & 10.9 & 12.6 & 12.5 \\
\hline 3 & $o$-Chlorophenyl & 73 & 59 & $\mathrm{C}_{9} \mathrm{H}_{7} \mathrm{~N}_{2} \mathrm{OSCl}$ & 12.5 & 12.4 & 14 & 14.1 \\
\hline 4 & p-Chlorophenyl & 112 & 72 & $\mathrm{C}_{9} \mathrm{H}_{7} \mathrm{~N}_{2} \mathrm{OSCl}$ & 12.1 & 12.4 & 14.2 & 14.1 \\
\hline 5 & $m$-Nitrophenyl & 152 & 65 & $\mathrm{C}_{9} \mathrm{H}_{7} \mathrm{~N}_{3} \mathrm{O}_{3} \mathrm{~S}$ & 17.5 & 17.7 & 13.4 & 13.5 \\
\hline 6 & $p$-Nitrophenyl & 198 & 69 & $\mathrm{C}_{9} \mathrm{H}_{7} \mathrm{~N}_{3} \mathrm{O}_{3} \mathrm{~S}$ & 17.4 & 17.7 & 13.3 & 13.5 \\
\hline 7 & Phenyl & 39 & 68 & $\mathrm{C}_{9} \mathrm{H}_{8} \mathrm{~N}_{2} \mathrm{OS}$ & 14.6 & 14.6 & 16.5 & 16.7 \\
\hline \multicolumn{9}{|c|}{$\mathbf{R}^{\prime}=$ Benzyl } \\
\hline 8 & o-Chlorophenoxymethyl & 108 & 69 & $\mathrm{C}_{16} \mathrm{H}_{13} \mathrm{~N}_{2} \mathrm{O}_{2} \mathrm{SCl}$ & 8.3 & 8.5 & 9.9 & 9.6 \\
\hline 9 & $m$-Chlorophenoxymethyl & 71 & 72 & $\mathrm{C}_{16} \mathrm{H}_{13} \mathrm{~N}_{2} \mathrm{O}_{2} \mathrm{SCl}$ & 8.7 & 8.5 & 9.8 & 9.6 \\
\hline 10 & $o$-Chlorophenyl & 58 & 65 & $\mathrm{C}_{15} \mathrm{H}_{11} \mathrm{~N}_{2} \mathrm{OSCl}$ & 9.1 & 9.2 & 10.4 & 10.6 \\
\hline 11 & p-Chlorophenyl & 118 & 74 & $\mathrm{C}_{15} \mathrm{H}_{11} \mathrm{~N}_{2} \mathrm{OSCl}$ & 9 & 9.2 & 10.7 & 10.6 \\
\hline 12 & $m$-Nitrophenyl & 158 & 64 & $\mathrm{C}_{15} \mathrm{H}_{11} \mathrm{~N}_{3} \mathrm{O}_{3} \mathrm{~S}$ & 13.1 & 13.4 & 10.1 & 10.2 \\
\hline 13 & $p$-Nitrophenyl & 201 & 70 & $\mathrm{C}_{15} \mathrm{H}_{11} \mathrm{~N}_{3} \mathrm{O}_{3} \mathrm{~S}$ & 13.7 & 13.4 & 10.4 & 10.2 \\
\hline 14 & Phenyl & 98 & 72 & $\mathrm{C}_{15} \mathrm{H}_{12} \mathrm{~N}_{2} \mathrm{OS}$ & 10.9 & 10.5 & 12 & 11.9 \\
\hline
\end{tabular}

was suspended in glacial acetic acid $(40 \mathrm{ml})$. To this was added $30 \%$ hydrogen peroxide $(12 \mathrm{ml})$ and the mixture was refluxed for $45 \mathrm{~min}$. Upon cooling and pouring the reaction mixture into water, a precipitate was obtained. This was filtered, washed with water and recrystallised from ethanol as a colourless solid mp $134^{\circ} \mathrm{C}$, yield $70 \%$ Found $\mathrm{C}, 48.4, \mathrm{H}, 3.6$; $\mathrm{C}_{9} \mathrm{H}_{8} \mathrm{~N}_{2} \mathrm{O}_{3} \mathrm{~S}$ required $\mathrm{C}, 48.2, \mathrm{H}, 3.6 \%$. Its IR spectrum reveals stretching vibration due to $-\mathrm{SO}_{2}-$ group at $1348 \sim 1337 \mathrm{~cm}^{-1}$, a medium band at 1433 and $1481 \mathrm{~cm}^{-1}$ (methyl group) and significant bands in the region 1560 and $1695 \mathrm{~cm}^{-1}$ (aromatic ring).

Other sulphones prepared by exactly the same pro- cedure are recorded in Table III.

Bis-(5-substituted-1, 3, 4-oxadiazol-2-yl) disulphides. An ethanolic solution of 2-mercapto oxadiazole compound $(0.02 \mathrm{M})$ was stirred with bromine $(0.04 \mathrm{M})$ in ethanol $(15 \mathrm{ml})$. The disulphide compound at once began to separate. It was filtered, washed and recrystallised if necessary. The disulphides thus prepared are recorded in Table $\mathrm{TV}$.

Fungicidal test. The test organisms were Aspergillus niger and Aspergillus flavus. The antifungal activity was evaluated by agar-growth technique at three different concentrations namely, $1: 1000,1: 10,000$ and 


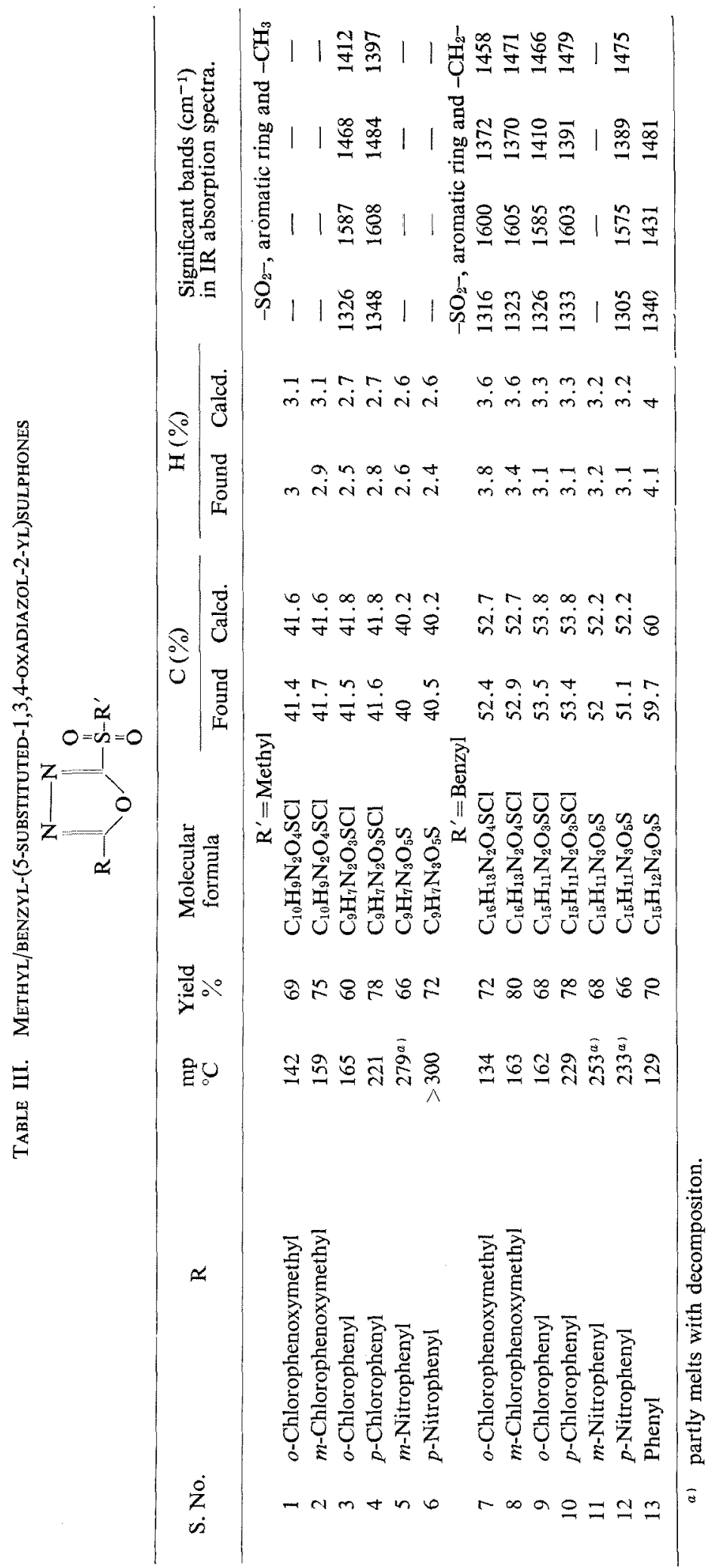


TABLE IV. BIS-(5-SUBSTITUTED-1,3,4-OXADIAZOL-2-YL)DISULPHIDES<smiles>[R]c1nnc(SSc2nnc([R])o2)o1</smiles>

\begin{tabular}{|c|c|c|c|c|c|c|c|c|}
\hline \multirow{2}{*}{ S.No. } & \multirow{2}{*}{$\mathrm{R}$} & \multirow{2}{*}{$\operatorname{mp}_{{ }^{\circ} \mathrm{C}}$} & \multirow{2}{*}{$\begin{array}{c}\text { Yield } \\
\%\end{array}$} & \multirow{2}{*}{$\begin{array}{l}\text { Molecular } \\
\text { formula }\end{array}$} & \multicolumn{2}{|c|}{$N(\%)$} & \multicolumn{2}{|c|}{$\mathrm{S}(\%)$} \\
\hline & & & & & Found & Calcd. & Found & Calcd. \\
\hline 10 & $o$-Chlorophenoxymethyl & 132 & 75 & $\mathrm{C}_{18} \mathrm{H}_{12} \mathrm{~N}_{4} \mathrm{O}_{4} \mathrm{~S}_{2} \mathrm{Cl}_{2}$ & 11.4 & 11.6 & 13.1 & 13.3 \\
\hline $2 n$ & m-Chlorophenoxymethyl & 151 & 80 & $\mathrm{C}_{18} \mathrm{H}_{12} \mathrm{~N}_{4} \mathrm{O}_{4} \mathrm{~S}_{2} \mathrm{Cl}_{2}$ & 11.5 & 11.6 & 13.6 & 13.3 \\
\hline 30 & o-Chlorophenyl & 157 & 70 & $\mathrm{C}_{16} \mathrm{H}_{8} \mathrm{~N}_{4} \mathrm{O}_{2} \mathrm{~S}_{2} \mathrm{Cl}_{2}$ & 13 & 13.2 & 15.4 & 15.1 \\
\hline $4 p$ & $p$-Chlorophenyl & 172 & 82 & $\mathrm{C}_{16} \mathrm{H}_{8} \mathrm{~N}_{4} \mathrm{O}_{2} \mathrm{~S}_{2} \mathrm{Cl}_{2}$ & 13.1 & 13.2 & 15 & 15.1 \\
\hline $5 n$ & $m$-Nitrophenyl & $278^{a 1}$ & 72 & $\mathrm{C}_{16} \mathrm{H}_{8} \mathrm{~N}_{6} \mathrm{O}_{6} \mathrm{~S}_{2}$ & 18.8 & 18.9 & 14.7 & 14.4 \\
\hline $6 p$ & $p$-Nitrophenyl & $292^{a 1}$ & 74 & $\mathrm{C}_{16} \mathrm{H}_{8} \mathrm{~N}_{6} \mathrm{O}_{6} \mathrm{~S}_{2}$ & 19.1 & 18.9 & 14 & 14.4 \\
\hline $7 \mathrm{~F}$ & Phenyl & $193 \sim 195$ & 82 & $\mathrm{C}_{16} \mathrm{H}_{10} \mathrm{~N}_{4} \mathrm{O}_{2} \mathrm{~S}_{2}$ & 16 & 15.8 & 18.4 & 18.1 \\
\hline
\end{tabular}

a) Partly melts with decomposition.

Table V. Antifungal Activity

\begin{tabular}{|c|c|c|c|c|c|c|c|}
\hline \multirow[b]{2}{*}{ S. No. } & \multirow[b]{2}{*}{ Compounds } & \multicolumn{6}{|c|}{ Average percentage inhibition after $96 \mathrm{hr}$} \\
\hline & & \multicolumn{3}{|c|}{$\begin{array}{c}\text { Organism-A. niger } \\
\text { Concentrations used } \\
1: 10001: 10,0001: 100,000\end{array}$} & \multicolumn{3}{|c|}{$\begin{array}{l}\text { Organism-A. flavus } \\
\text { Concentrations used } \\
1: 10001: 10,0001: 100,000\end{array}$} \\
\hline 1 & $\begin{array}{l}\text { Methyl-[5-(o-chlorophenoxymethyl)- } \\
\text { 1,3,4-oxadiazol-2-yl]sulphone }\end{array}$ & 68.2 & 62.4 & 50.1 & 65.3 & 59.2 & 40.6 \\
\hline 2 & $\begin{array}{l}\text { Benzyl-[5-(o-chlorophenoxymethyl)- } \\
\text { 1,3,4-oxadiazol-2-yl]sulphone }\end{array}$ & 62.4 & 56.1 & 41 & 56.2 & 49 & 32.4 \\
\hline 3 & $\begin{array}{l}\text { Benzyl-[5-(m-chlorophenoxymethyl)- } \\
\text { 1,3,4-oxadiazol-2-yl]sulphone }\end{array}$ & 89.6 & 76.8 & 42.2 & 88.4 & 70.2 & 38.7 \\
\hline 4 & $\begin{array}{l}\text { Methyl-[5-(o-chlorophenyl)- } \\
\text { 1,3,4-oxadiazol-2-yl]sulphone }\end{array}$ & 78 & 69.2 & 50.5 & 62 & 50.2 & 42 \\
\hline 5 & $\begin{array}{l}\text { Benzyl-[5-(o-chlorophenyl)- } \\
\text { 1,3,4-oxadiazol-2-yl]sulphone }\end{array}$ & 66.4 & 60 & 49.2 & 67.4 & 51 & 39.4 \\
\hline 6 & $\begin{array}{l}\text { Benzyl-[5-( } p \text {-chlorophenyl })- \\
1,3,4 \text {-oxadiazol-2-yl]sulphone }\end{array}$ & 71 & 62.6 & 52.4 & 68.5 & 51.2 & 42 \\
\hline 7 & $\begin{array}{l}\text { Methyl-[5-(m-nitrophenyl)- } \\
\text { 1,3,4-oxadiazol-2-yl]sulphone }\end{array}$ & 63.2 & 58.1 & 41.9 & 66.6 & 65.4 & 43.4 \\
\hline 8 & $\begin{array}{l}\text { Benzyl-[5-( } p \text {-nitrophenyl }) \text { - } \\
\text { 1,3,4-oxadiazol-2-yl]sulphone }\end{array}$ & 66.5 & 57 & 42.6 & 64.2 & 58 & 41 \\
\hline 9 & $\begin{array}{l}\text { 5-( } p \text {-chlorophenyl)-2-mercapto- } \\
1,3,4 \text {-oxadiazole }\end{array}$ & 70 & 66.8 & 50 & 68.4 & 59 & 38.4 \\
\hline 10 & $\begin{array}{l}\text { 5-(o-chlorophenyl)-2-mercapto- } \\
\text { 1,3,4-oxadiazole }\end{array}$ & 65.6 & 59.3 & 39 & 69.4 & 58.4 & 32.3 \\
\hline 11 & $\begin{array}{l}\text { 5-(m-chlorophenoxymethyl)-2- } \\
\text { mercapto-1,3,4-oxadiazole }\end{array}$ & 80 & 60.6 & 40.4 & 79 & 62.5 & 38.7 \\
\hline 12 & $\begin{array}{l}\text { Methy]-[5-(m-chlorophenoxymethyl)- } \\
\text { 1,3,4-oxadiazol-2-yl]sulphide }\end{array}$ & 70.2 & 56.2 & 35 & 60.4 & 51 & 31.5 \\
\hline 13 & $\begin{array}{l}\text { Benzyl-[5-(p-chlorophenoxymethyl)- } \\
1,3,4 \text {-oxadiazol-2-yl]sulphide }\end{array}$ & 70.2 & 56.2 & 35 & 60.4 & 51 & 31.5 \\
\hline 14 & $\begin{array}{l}\text { Bis-[5-( } p \text {-chlorophenyl)- } \\
1,3,4 \text {-oxadiazol-2-yl]disulphide }\end{array}$ & 67.3 & 52.3 & 43.4 & 68.2 & 55 & 41.6 \\
\hline 15 & $\begin{array}{l}\text { Bis-[5-(o-chlorophenoxymethyl)- } \\
1,3,4 \text {-oxadiazol-2-yl]disulphide }\end{array}$ & 72.4 & 51.6 & 46.6 & 70.9 & 50.3 & 36.2 \\
\hline
\end{tabular}


1: 100,000 and number of replications in each case was three. The average percentage inhibition by various compounds is recorded in Table $\mathrm{V}$.

\section{RESULTS AND DISCUSSION}

In view of the reported pesticidal properties of a variety of sulphones. ${ }^{5 \sim 8)}$ it was considered worthwhile to study the fungicidal properties of oxadiazolyl sulphones and sulphides. Obviously these compounds are fungitoxic at higher concentration but their toxicity decreases upon dilution. Further, their activity varies with the species of fungus as well.

The mercapto oxadiazole (compound No. 9) having a $o$-chlorophenyl substituent is more active against $A$. niger than $A$. flavus. The compound Nos. 1, 4, 5, 6, 7, 8 and 14 which are representatives of sulphides and sulphones appear to be promising fungicides. It is noteworthy that all of them have either a chlorophenyl or nitrophenyl substituent.

These results are in conformity with earlier observations that heterocyclic rings with aryl or other lipophilic substituents are usually fungitoxic.
Acknowledgement. The authors express their sincere thanks to Dr. R. P. Rastogi, Professor and Head of the Chemistry Department, University of Gorakhpur, Gorakhpur, U.P. (India) for providing necessary facilities.

\section{REFERENCES}

1) G. Werber, M. C. Aversa and F. Buccheri, Ann. Chim (Rome), 59(10), 912 (1969).

2) Tohoku Yakka Daigaku Kenkyu Nempo, 17, 43 (1970).

3) S. Umio, K. Karyone and T. Kishimoto, Japan, 7006,266 (Cl. 16E 391.2).

4) Y. Okada, Japan 7024, 982 (Cl. 16E 391.2).

5) Branes, J. Econ. Entomol, 44, 672 (1951).

6) Kirdy and Tew, Rep. Prog. Appl. Chem., 37, 263 (1952).

7) N. V. Philips, Gloeilampenfabrieken, Dutch 81 , 359, May 15, 1956.

8) U. S. Pat. 2,645, 592 (1953).

9) Mays, "Chemistry of Synthetic drugs," 5th Ed., p. 602 .

10) L. L. Bambas, I. Amer. Chem. Soc., 67, 688 (1945).

11) Horsfall and Rich, Cont. Boyc. Thompson, Institute, 16, 346 (1951).

12) R. W. Young and K. H. Wood, J. Amer. Chem. Soc., 77, 400 (1955).

13) F. Russo and M. Ghelardoni, Boll. Chim. Farm., 106, 826 (1967).

14) O. Turilli and M. Gandino, Ann. Chim. (Rome), 53(11), 1687 (1963). 